\title{
Valoración del maíz como patrimonio natural por mujeres amas de casa
}

\section{Valuation of Corn as a Natural Heritage by Women Housewives}

\section{Avaliação do milho como patrimônio natural de mulheres donas de casa}

Rafael Manuel de Jesús Mex-Álvarez

Universidad Autónoma de Campeche, Facultad de Ciencias Químico Biológicas, México rafammex@uacam.mx https://orcid.org/0000-0003-1154-0566

Patricia Margarita Garma-Quen

Universidad Autónoma de Campeche, Facultad de Ciencias Químico Biológicas, México pamgarma@uacam.mx https://orcid.org/0000-0003-4347-0347

David Yanez-Nava

Universidad Autónoma de Campeche, Facultad de Ciencias Químico Biológicas, México davyanez@uacam.mx https://orcid.org/0000-0001-9604-526X 


\section{Revista Iberoamericana \\ de las Ciencias Sociales y \\ Humanísticas}

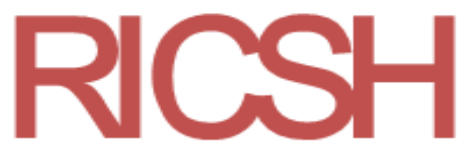

ISSN: $2395-7972$

\section{Resumen}

El maíz es patrimonio del pueblo de México, pues es un elemento identitario de su cultura y sociedad y es un ingrediente indispensable en la gastronomía mexicana. El objetivo de esta investigación fue conocer los valores y la sensibilidad al cambio de las dimensiones cultural, económica, salud y social del maíz. Se aplicó un cuestionario validado a 537 mujeres en mercados y supermercados de San Francisco de Campeche, México, para conocer su opinión sobre la importancia sociocultural, económica y alimentaria del maíz. Se encontró que los valores existentes en el uso y consumo del maíz muestran una mayor correlación con la edad. Las mujeres más jóvenes presentan una menor magnitud en las dimensiones evaluadas. Todas las generaciones, sin embargo, exhiben una intensidad alta a la sensibilidad al cambio. Esto representa una necesidad de educar respecto a la importancia de la agrodiversidad del maíz para su defensa como patrimonio natural.

Palabras clave: agrodiversidad, alimento funcional, gastronomía.

\section{Abstract}

Corn is the heritage of the Mexican people. It is an identity element of its culture and society and is an indispensable ingredient in its cuisine. The objective of this research was to know the values and sensitivity to change of the cultural, economic, health and social dimensions of corn. A validated questionnaire was applied to 537 women who buy their food to get their opinion on the socio-cultural, economic and food importance of corn. It was found that existing values in the use and consumption of corn show a greater correlation with age. Younger women have a lower magnitude in the dimensions of values evaluated. However, all generations exhibit a high intensity of sensitivity to change. This represents a need to educate about the importance of corn agrodiversity for its defense as heritage natural.

Keywords: agrodiversity, functional food, gastronomy. 


\section{Revista Iberoamericana \\ de las Ciencias Sociales y \\ Humanísticas}

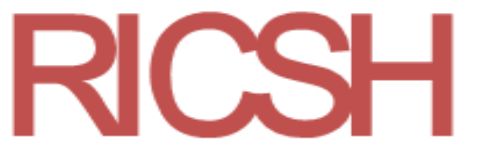

ISSN: $2395-7972$

\section{Resumo}

O milho é uma herança do povo do México, pois é um elemento de identidade de sua cultura e sociedade e é um ingrediente indispensável na gastronomia mexicana. O objetivo desta pesquisa foi conhecer os valores e a sensibilidade à mudança das dimensões cultural, econômica, sanitária e social do milho. Um questionário validado foi aplicado a 537 mulheres em mercados e supermercados em San Francisco de Campeche, México, para descobrir sua opinião sobre a importância sociocultural, econômica e alimentar do milho. Verificou-se que os valores existentes no uso e consumo de milho apresentam maior correlação com a idade. Mulheres mais jovens têm uma magnitude menor nas dimensões avaliadas. Todas as gerações, no entanto, exibem uma alta intensidade de sensibilidade à mudança. Isso representa uma necessidade de educar sobre a importância da agro-diversidade do milho para sua defesa como patrimônio natural.

Palavras-chave: agrodiversidade, comida funcional, gastronomia.

Fecha Recepción: Diciembre 2019

Fecha Aceptación: Julio 2020

\section{Introducción}

La alimentación es un proceso biopsicosocial íntimamente relacionado con el ser humano. Sin duda es un fenómeno complejo. Aunque la sensación de hambre se origina a nivel fisiológico, hay elementos psicológicos que influyen en la selección de los alimentos, ya sea por sus propiedades organolépticas agradables, oportunidad en relación con las condiciones ambientales o por recuerdos afectivos, y en la satisfacción a la hora de comer. El componente social de la alimentación se constata a través de la historia, como eje de la vida social y base de la cohesión de grupos familiares y de amistad que incluyen eventos sociales y culturales (Reynoso, González y Salgado, 2007; Sánchez y Cortez, 2006). En ese sentido, a pesar de la diversidad étnica y geográfica, la gastronomía mexicana contiene como elementos comunes en todo el territorio el consumo de maíz, frijol, calabaza y chile, al igual que la costumbre de preparar antojitos (Altieri, 2004; Brutus, 2007; Sánchez y Cortez, 2006; Sangerman, Acosta, Schwenstesius de Rindermann, Damián y Larqué 2010). La cultura gastronómica mexicana se originó 2000 años antes de la llegada del imperio español y se enriqueció a través de los siglos (D’Alessandro y González, 2017; Espinosa et al., 2003). 


\section{Revista Iberoamericana \\ de las Ciencias Sociales y \\ Humanísticas}

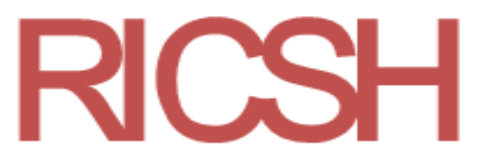

ISSN: 2395 - 7972

La riqueza sociocultural implicada en la gastronomía mexicana originó que fuera propuesta en 2002 para formar parte del patrimonio cultural inmaterial de la humanidad y sea protegida por la Organización de las Naciones Unidas para la Educación, Ciencia y Cultura (Unesco). En noviembre de 2010 se aceptó la nominación y se determinó que la gastronomía mexicana debe ser preservada y protegida por todos los mexicanos, fundamentalmente por todas las instancias de Gobierno por ser parte de la riqueza cultural del país, por sus características originales que pueden estar en peligro de desaparecer y para hacer frentes a los peligros como la competencia de cadenas transnacionales, sean de comida rápida o de transgénicos (Arellano y Arriaga, 2001; Brutus, 2007; Castro, 2005; Sánchez y Cortez, 2006). Por ello, el objetivo del presente trabajo de investigación fue determinar los valores que las mujeres amas de casa le dan al maíz como elemento esencial de la gastronomía, cultura, economía y sociedad mexicana. Esto para identificar cuáles son las motivaciones que permitirían proteger y defender al maíz como patrimonio natural de México.

\section{Metodología}

Para la valoración del maíz como alimento patrimonio natural se utilizó un tipo de estudio observacional transversal. Primero se realizaron entrevistas domiciliarias y luego encuestas levantadas en cinco mercados y 13 supermercados a mujeres amas de casa que frecuentan estos espacios de la ciudad de San Francisco de Campeche, México. Inicialmente se diseñó un cuestionario dividido en dos partes y cuatro categorías. La primera parte versaba sobre los valores existentes y la segunda sobre la sensibilidad al cambio de dichos valores; cada parte se agrupó en cuatro categorías de valores: económica, cultural, social y salud (biológica). Cada categoría contenía cuatro ítems. Cada ítem representaba un valor (con un total de 32 ítems). Había cinco opciones de respuesta frente a cada valor: Muy de acuerdo (MA), De acuerdo (A), Indiferente (o no sabe) (I), En desacuerdo (D) y Muy en desacuerdo (MD). Estas opciones representaban una escala de puntuación de Likert $(\mathrm{MA}=+2, \mathrm{~A}=+1$, $\mathrm{I}=0, \mathrm{D}=-1, \mathrm{MD}=-2$ ). El cuestionario fue validado y posteriormente se aplicó a una población de 537 mujeres. El sentido de los valores se obtuvo por la suma de los valores de las respuestas $(\mathrm{S})$ proporcionadas y la intensidad del valor se calculó por la suma del cuadrado de los valores (C) de las respuestas. A manera de ejemplificación, el proceso de asignación de valores numéricos se muestra en la tabla 1. 


\section{Revista Iberoamericana \\ de las Ciencias Sociales y \\ Humanísticas}

ISSN: $2395-7972$

Tabla 1. Ejemplo de asignación de puntaje de los ítems de una categoría

\begin{tabular}{|c|r|c|c|c|c|c|c|c|}
\hline & MD & D & I & A & MA & S & C \\
\hline 1 & $\begin{array}{c}\text { Consumiría otras variedades de maíz (morado, } \\
\text { rojo, azul) como alimento. }\end{array}$ & $\checkmark$ & & & & & -2 & +4 \\
\hline 2 & $\begin{array}{c}\text { Debe enseñarse a los niños y jóvenes sobre la } \\
\text { importancia del maíz. }\end{array}$ & & & $\checkmark$ & & & 0 & 0 \\
\hline 3 & $\begin{array}{r}\text { Hay que promover los usos culturales } \\
\text { (tradicionales) del maíz. }\end{array}$ & & & & $\checkmark$ & & +1 & +1 \\
\hline 4 & $\begin{array}{c}\text { Defendería al maíz como patrimonio de la } \\
\text { humanidad. }\end{array}$ & & $\checkmark$ & & & & -1 & +1 \\
\hline & Total & & & & & & -2 & +6 \\
\hline
\end{tabular}

Fuente: Elaboración propia

\section{Resultados}

Los datos sociodemográficos de la población encuestada se reportan en la tabla 2 y tabla 3. En ellas se puede observar que el rango que concentra la mayor parte de la población encuestada está entre los 20 y 40 años. Además, el nivel de estudio de secundaria, preparatoria y licenciatura fueron los más comunes.

Tabla 2. Distribución de las edades de las mujeres que contestaron el cuestionario

\begin{tabular}{|c|c|c|}
\hline Grupo etario & Número (porcentaje) & Edad promedio \\
\hline En los 20 & $161(29.98 \%)$ & 27 \\
\hline En los 30 & $100(18.62 \%)$ & 34 \\
\hline En los 40 & $148(27.75 \%)$ & 46 \\
\hline En los 50 & $84(15.64 \%)$ & 53 \\
\hline En los 60 & $43(8.01 \%)$ & 66 \\
\hline
\end{tabular}

Fuente: Elaboración propia 


\section{Revista Iberoamericana \\ de las Ciencias Sociales y \\ Humanísticas}

ISSN: $2395-7972$

Tabla 3. Distribución del nivel de estudio de las mujeres entrevistadas

\begin{tabular}{|c|c|c|c|c|c|}
\hline Grupo etario & Primaria & Secundaria & Preparatoria & Licenciatura & Postgrado \\
\hline En los 20 & 0 & 13 & 67 & 81 & 0 \\
\hline En los 30 & 8 & 42 & 17 & 33 & 0 \\
\hline En los 40 & 17 & 67 & 34 & 29 & 2 \\
\hline En los 50 & 16 & 35 & 14 & 19 & 0 \\
\hline En los 60 & 8 & 12 & 16 & 6 & 1 \\
\hline
\end{tabular}

Fuente: Elaboración propia

Estas entrevistas se realizaron en los lugares donde se compran alimentos, es decir, in situ. La descripción detallada del número de entrevistas realizadas en cada establecimiento y su ubicación dentro de la ciudad se muestra en la tabla 4 y figura 1, respectivamente.

Tabla 4. Relación de mercados y supermercados donde se entrevistaron a las mujeres

\begin{tabular}{|c|c|c|c|c|c|c|c|c|}
\hline & Mercado & $\#$ & & Supermercado & $\#$ & & Supermercado & $\#$ \\
\hline $\mathbf{1}$ & Central & 98 & A & Soriana CTM & 19 & H & San Fco. & 24 \\
\hline $\mathbf{2}$ & Ejidal & 17 & $\mathbf{B}$ & Chedraui E. & 24 & I & Walmart & 18 \\
\hline $\mathbf{3}$ & San Fco. & 49 & $\mathbf{C}$ & Soriana Kala & 21 & J & Comercial & 25 \\
\hline $\mathbf{4}$ & Concordia & 26 & $\mathbf{D}$ & Aurrera G & 22 & $\mathbf{K}$ & Express F & 19 \\
\hline $\mathbf{5}$ & Fidel & 28 & $\mathbf{E}$ & Express C & 15 & L & Aurrera R & 22 \\
\hline $\mathbf{6}$ & Morelos & 21 & $\mathbf{F}$ & Chedraui G & 25 & $\mathbf{M}$ & Soriana U & 28 \\
\hline & & & $\mathbf{G}$ & SAMS & 15 & $\mathbf{N}$ & Aki Ría & 21 \\
\hline & Total & $\mathbf{2 3 9}$ & & & & & Total & $\mathbf{2 9 8}$ \\
\hline
\end{tabular}

Fuente: Elaboración propia 


\section{Revista Iberoamericana \\ de las Ciencias Sociales y \\ Humanísticas}

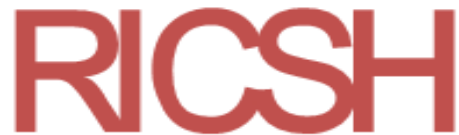

ISSN: $2395-7972$

Figura 1. Mapa de la ciudad de Campeche que muestran los mercados (azul) y supermercados (amarillo) donde se realizaron las entrevistas

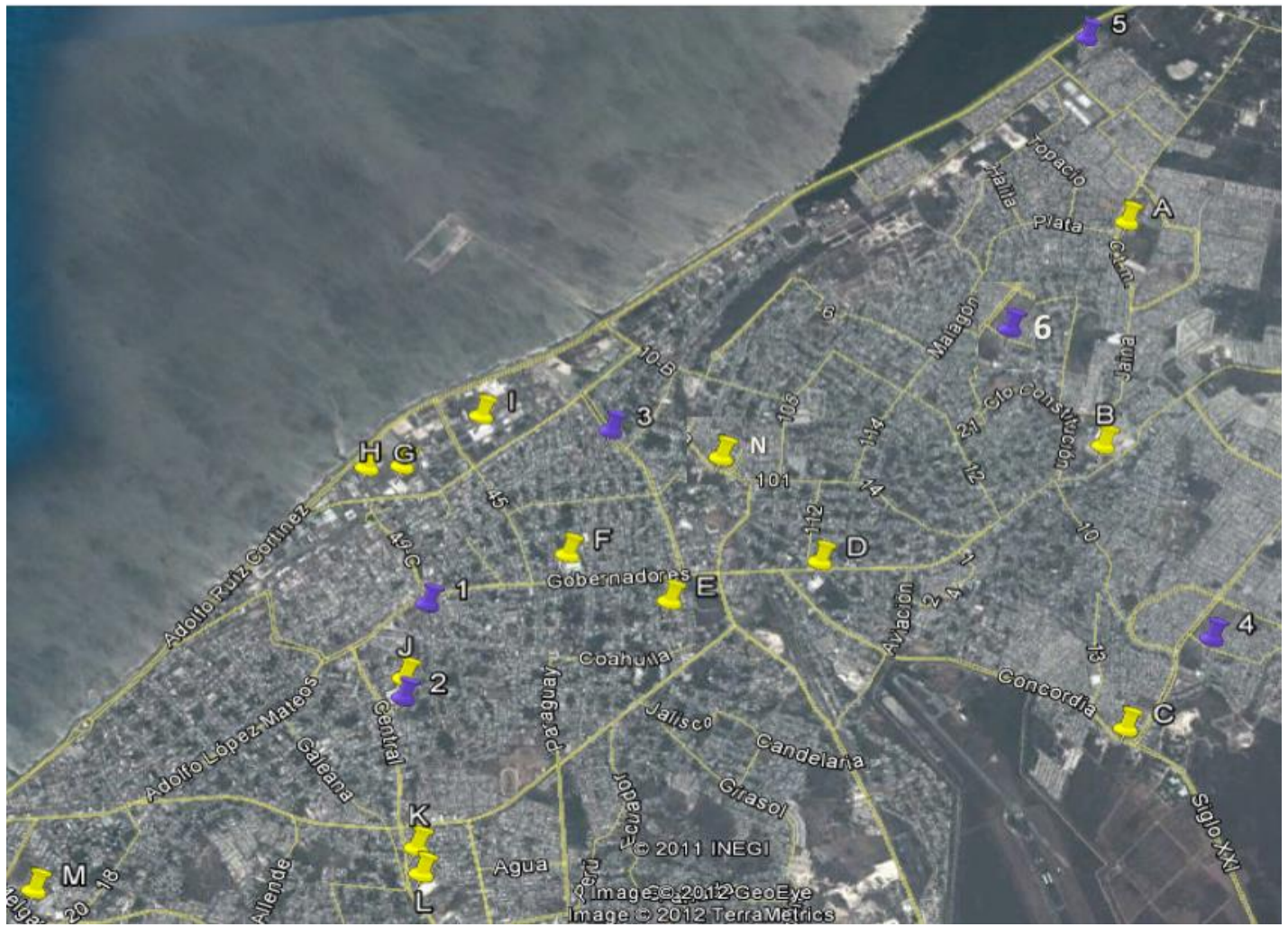

Fuente: Elaboración propia con base Google Maps

En la figura 2 se muestra una tendencia similar en los distintos grupos etarios que, en general, define una marcada deficiencia en los valores económicos y una presencia sustantiva de valores culturales, sociales y sanitarios, que se van consolidando a través de las generaciones, es decir, que las amas de casa, aunque se preocupan por el precio del maíz, no lo consideran un factor importante para dejar de consumirlo y ponderan más el carácter identitario del maíz como mexicanos y lo consideran un elemento indispensable en la gastronomía. Considerando todo el universo de entrevistadas, se puede observar que la principal preocupación y mayor valoración es hacia el componente de salud, seguido de los componentes sociales y culturales con una magnitud equiparable; en último lugar se ubicó el valor económico. 


\section{Revista Iberoamericana \\ de las Ciencias Sociales y \\ Humanísticas}

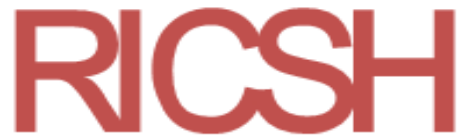

ISSN: $2395-7972$

Figura 2. Sentido de la valorización del maíz como patrimonio natural por mujeres

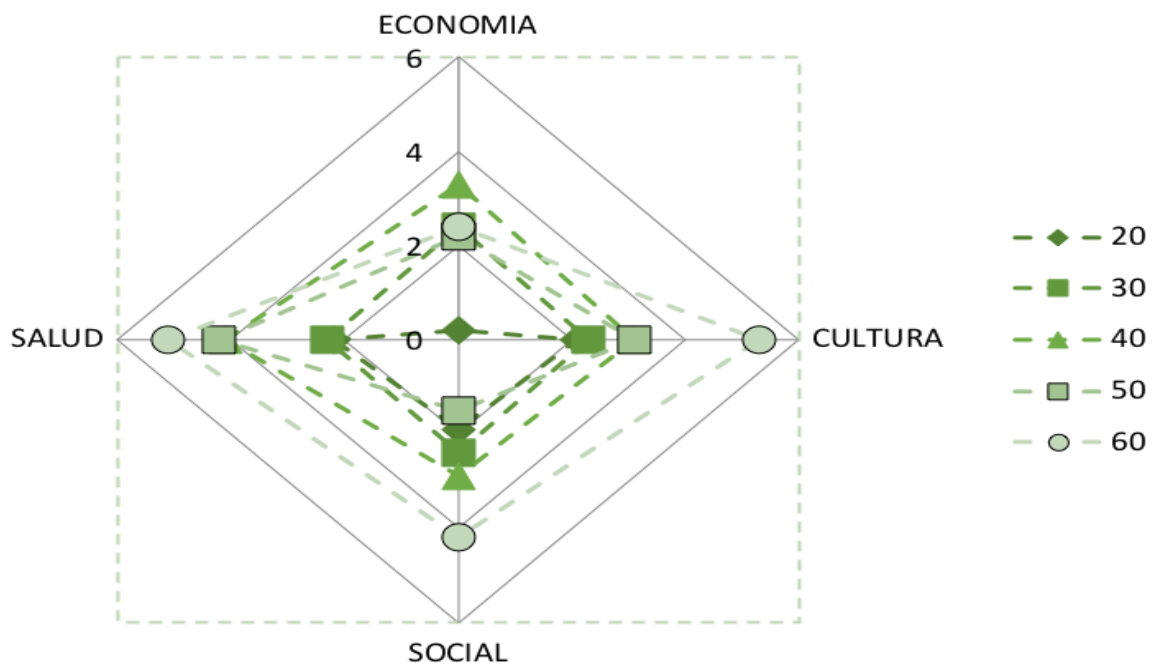

Fuente: Elaboración propia

Figura 3. Intensidad de la valorización del maíz por mujeres

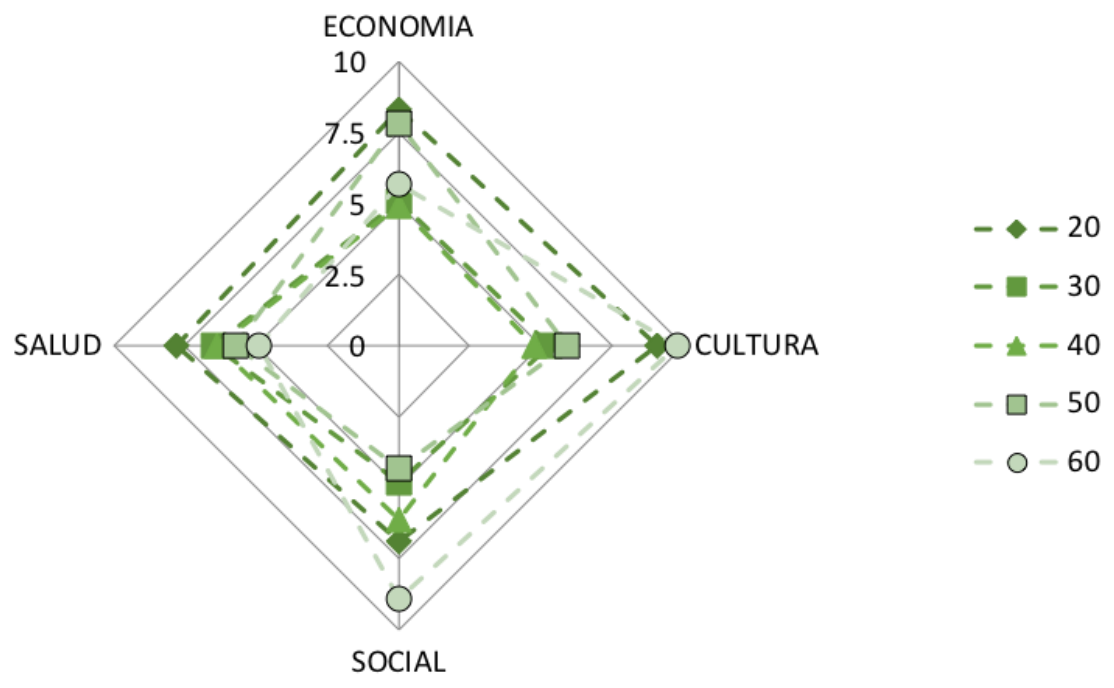

Fuente: Elaboración propia

Al analizar la gráfica de intensidad de los valores de la figura 3, se puede contrastar que, a pesar de que el sentido del valor de salud destaca entre los demás factores y se pondera más positivamente que otros, la intensidad de los valores es muy similar en los cuatro rubros en cada grupo generacional. En contraste, el conjunto de datos obtenidos en todos los grupos parece sugerir que el valor sanitario es el que posee la menor intensidad y los valores culturales, económicos y sociales tienen una mayor intensidad respecto al sanitario, pero 


\section{Revista Iberoamericana \\ de las Ciencias Sociales y \\ Humanísticas}

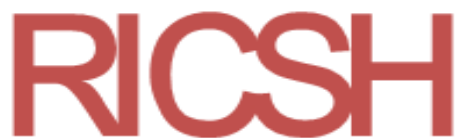

ISSN: 2395 - 7972

semejantes entre sí, aunque existe una ligera tendencia de una mayor intensidad de los valores culturales. En esa gráfica también se observa una mayor uniformidad de distribuciones que pareciera agrupar las generaciones en dos clases, aunque en realidad no pudiera hacerse dicha agrupación por la heterogeneidad en las decisiones. Así, en el rubro de salud la mayor intensidad se presentó en quienes se ubicaban en sus 20; en referencia a la economía, quienes estaban en los 20 y los 50 indicaron una mayor intensidad respecto al resto; los grupos etarios de 20 y 60 años exhibieron una mayor magnitud por la cultura, y los sexagenarios mostraron una mayor ponderación del aspecto social. Asimismo, las figuras 4 y 5 representan el sentido y la intensidad, respectivamente, de la sensibilidad al cambio de la valoración del maíz por mujeres amas de casa.

Figura 4. Sentido de la sensibilidad de los valores al cambio del maíz como patrimonio

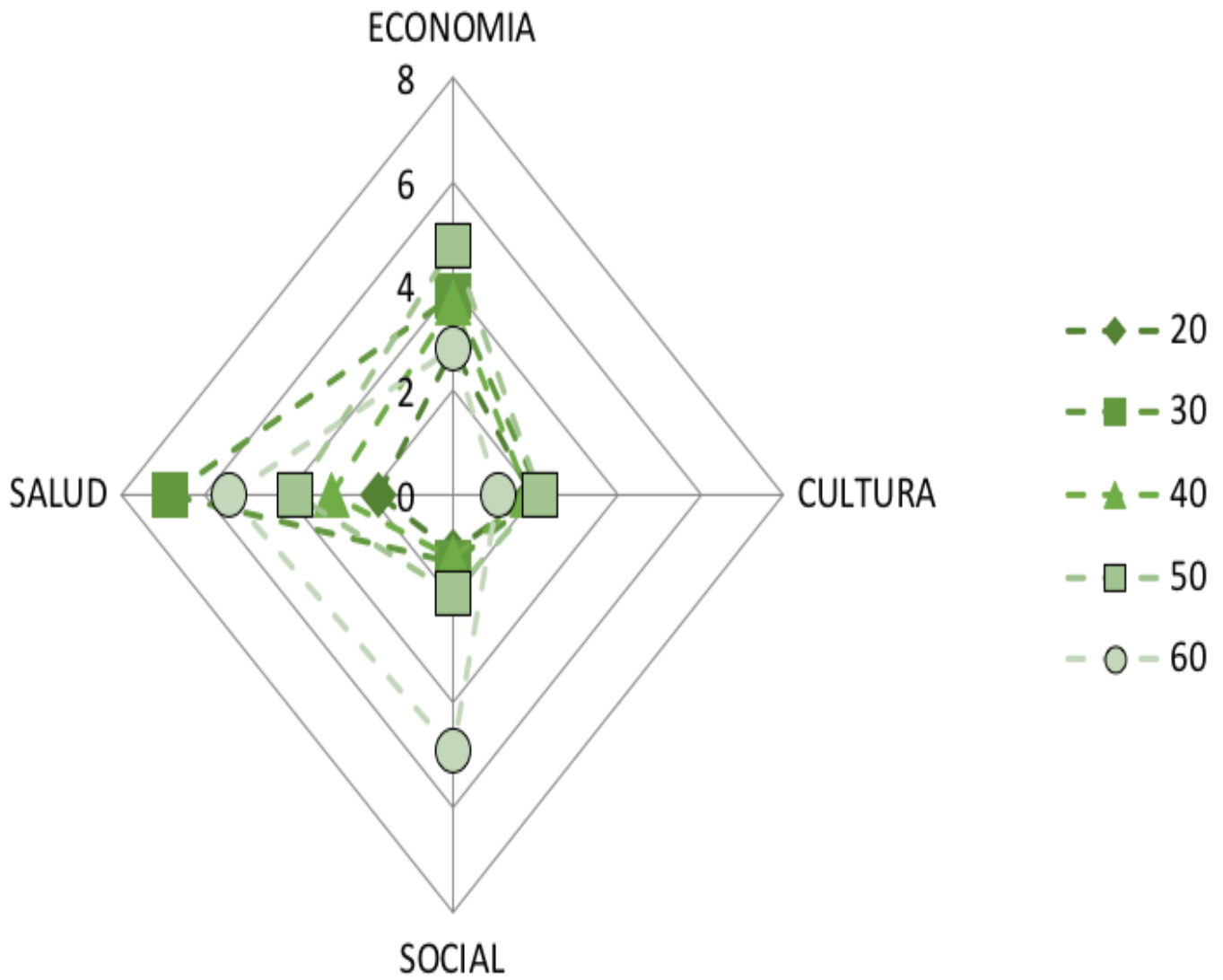

Fuente: Elaboración propia 


\section{Revista Iberoamericana \\ de las Ciencias Sociales y \\ Humanísticas}

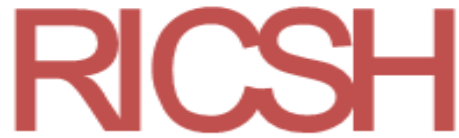

ISSN: $2395-7972$

Figura 5. Intensidad de la sensibilidad de los valores del maíz como patrimonio

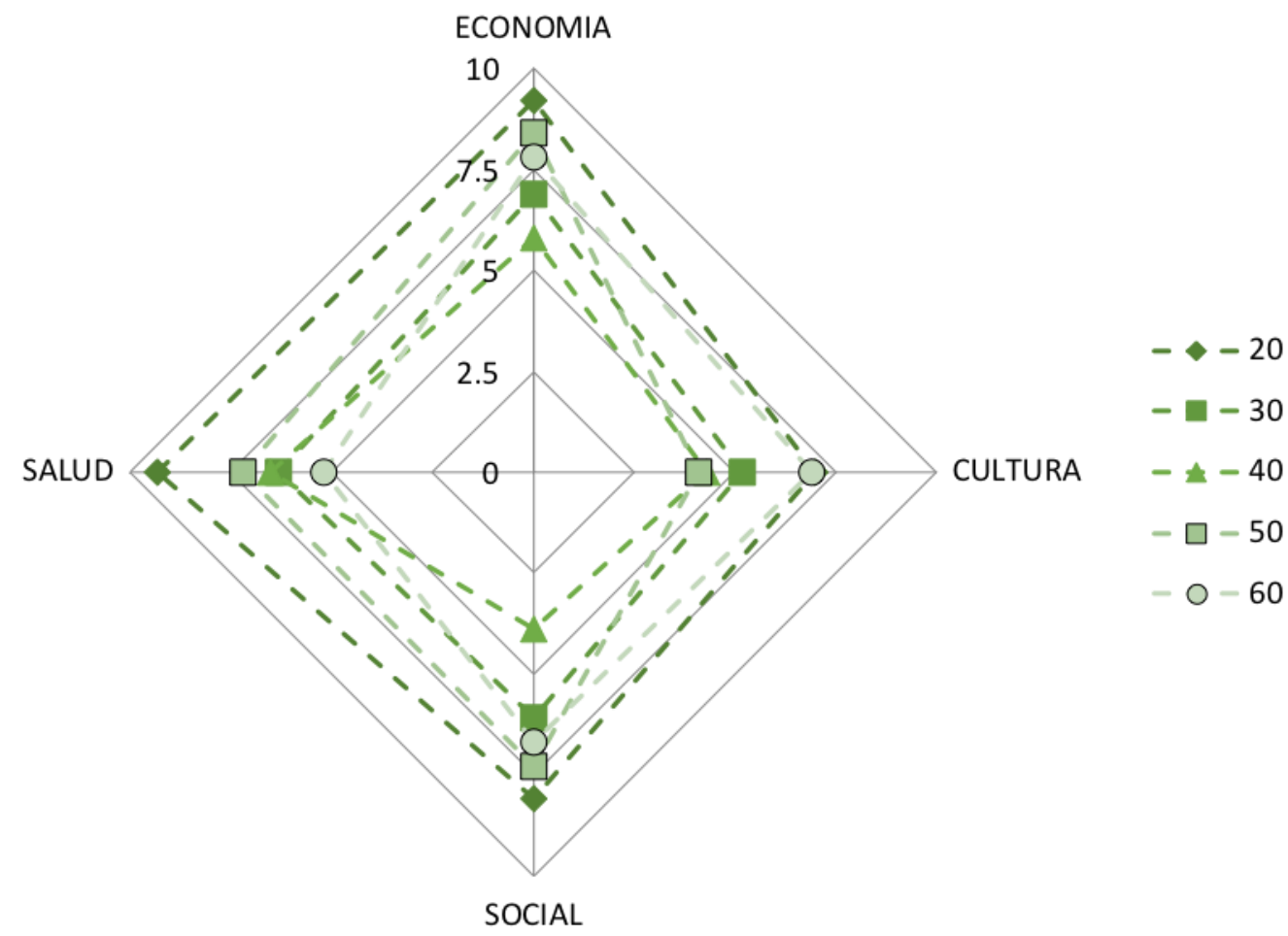

Fuente: Elaboración propia

Los resultados obtenidos demuestran que existe una mayor preocupación por la relación de la alimentación a base de maíz con la salud que respecto a los otros aspectos como punto de mejora o de mayores expectativas y oportunidades, incluso de mayor magnitud que la economía; de hecho, los valores culturales muestran la menor fuerza al cambio. Por su parte, la intensidad de los valores al cambio muestra una distribución similar entre todos los grupos etarios, con una intensidad similar entre los cuatro rubros, con una ligera tendencia mayor hacia el aspecto sanitario y una intensidad ligeramente menor respecto a lo cultural. 


\section{Revista Iberoamericana \\ de las Ciencias Sociales y \\ Humanísticas}

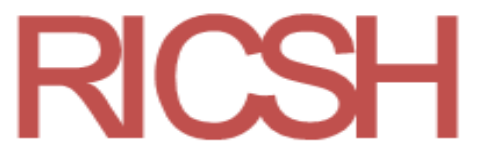

ISSN: $2395-7972$

\section{Discusión}

Para la valoración del maíz como patrimonio natural se realizó una investigación social basada en metodologías similares para identificar y jerarquizar valores. Antes de elaborar las encuestas para reconocer la opinión general y empezar a ubicar los valores que se presentan en la población femenina, se realizaron entrevistas con el fin de recopilar la mayor información posible y evitar sesgos importantes. El ciclo de sustentabilidad se reinicia nuevamente al estimar la percepción de la población y jerarquizar y cuantificar los valores existentes en relación con el uso y consumo del maíz como alimento y su sensibilidad ante el planteamiento del maíz como patrimonio natural que lo posiciona como una opción sustentable (Rodríguez, Chávez, Thomé y Miranda, 2017).

Los resultados de la valoración demuestran una heterogeneidad, especialmente respecto al aspecto económico; las mujeres veinteañeras, por mencionar un ejemplo, registraron una escasa valoración de este. Los valores culturales y sociales exhiben un comportamiento similar entre todos los grupos de edad, aunque hay una mayor acentuación en las sexagenarias. Y el comportamiento de los valores sanitarios presenta una tendencia a la alza conforme aumenta la edad; así, las mujeres de 20 y 30 años de edad presentan menor interés por su salud o seguridad sanitaria y el resto de los grupos etarios muestran un incremento de preocupación e interés por este aspecto: cuando transcurren los años se hace más frecuente y probable la aparición y el padecimiento de enfermedades crónico degenerativas, cáncer y otros problemas de salud que obligan a tener más conciencia. Las personas más jóvenes se preocupan más del aspecto estético relacionado con la alimentación en vez del saludable; por ello, en sus respuestas advertimos un interés en el alto contenido de azúcar del maíz asociado a la creencia de que engorda, aunado a la ignorancia de sus propiedades medicinales, y tienden a consumir alimentos ligeros, bajos en calorías, saludables e integrales sin discernir claramente entre el significado de cada concepto anterior y menos aplicarlos prácticamente (Fernández, Morales y Gálvez, 2013; Garza y Cantú, 2002).

No obstante lo anterior, el análisis de la intensidad de los valores muestra intereses similares entre las generaciones. Esto indica que la ausencia de valores registrada en los datos anteriores no se debe a una indiferencia ante el maíz y los problemas socioculturales, económicos y sanitarios; por el contrario, se debe a la coexistencia de principios, valores e ideas positivas junto con pesimismo, desilusión, apatía o confusión que genera errores e 


\section{Revista Iberoamericana \\ de las Ciencias Sociales y \\ Humanísticas}

ISSN: $2395-7972$

ignorancia. Contrariamente a lo que pudiera deducirse del análisis anterior, las veinteañeras exhibieron una intensidad muy alta en todos los aspectos: tuvieron la mayor intensidad en el aspecto de valores de salud y sociales, la mayor intensidad junto con las mujeres de cincuenta años en el ámbito económico y la segunda intensidad más alta equivalente a la intensidad de las sexagenarias respecto a los valores culturales.

Lo anterior permite sugerir que las generaciones más jóvenes no carecen del todo de valores, sino que se encuentran en un proceso de definición o maduración. Como no existe indiferencia sino inconformidad, esto permite aprovechar el interés de las mujeres más jóvenes para formarlas y fortalecer la defensa del patrimonio y promoción del cuidado de la salud. La educación de este sector haría que se convierta en un público más demandante y exigente de bienes y servicios de mejor calidad, en mayor variedad, con mejores ofertas, seguros, confiables y dignos, que se convertiría en una fuerte presión política para obligar a recapacitar a los tomadores de decisiones y generar políticas públicas, leyes y acciones en beneficio del campo y de la seguridad alimentaria de todos los mexicanos y defender al maíz como patrimonio de la humanidad y legado del pueblo de México (Olivé, 2009; Rodríguez et al., 2017).

Identificar al maíz como logro (en lo referente a su domesticación, conservación y diversificación) de los mexicanos, apreciar su variedad y los beneficios de la agrodiversidad del campo mexicano y contrastarlo con los peligros del uso de transgénicos e híbridos, los riesgos e impactos a la salud, medio ambiente y biodiversidad garantizaría la defensa por parte del pueblo del maíz y otros productos agrícolas. Al respecto, es conveniente comentar que uno de los argumentos que se proponía a las mujeres fue la frase "Sin maíz no hay país", lema de una de las asociaciones comprometidas con la defensa del maíz mexicano, y contrario a la hipótesis que suponía un grado concordancia (MA o A) con la frase, una cantidad importante de mujeres, generalmente las jóvenes, comentó estar en desacuerdo $(21 \%)$ o en total desacuerdo (9\%) con ella y otras más demostró indiferencia (16\%).

La explicación frecuente a esto fue que existe también el trigo y otros cereales, por si el enunciado se refería al maíz como alimento; otras criticaron la importancia del maíz como un producto no indispensable en la alimentación o que puede ser sustituido; también algunas

dijeron que si se acabara el maíz el país seguiría porque la sociedad, la economía, la tecnología no depende del maíz. Estas posturas son realmente alarmantes y sugieren una intervención expedita para contrarrestarlas, porque la contaminación en el caso del maíz, muy 


\section{Revista Iberoamericana \\ de las Ciencias Sociales y \\ Humanísticas}

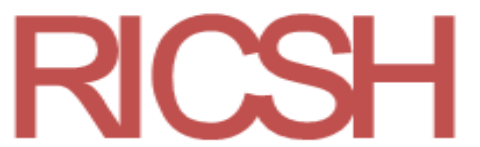

ISSN: 2395 - 7972

aparte del aspecto biológico, incluye igualmente una contaminación de las ideas y conciencias que provocan una reacción en sentido contrario y radicalmente opuesta a la protección del patrimonio y su conservación.

Para saber qué estrategias seguir o cómo abordar el problema, resulta útil conocer qué espera la gente o a qué valores son más sensibles para fijar un punto de partida e iniciar las actividades integrales de educación y acción ciudadana. En este caso, las mujeres fueron más sensibles a los valores de salud, es decir, tienen una mayor expectativa a una mejor oferta en aspectos relacionados con la conservación del peso, disminución de riesgos por contaminación microbiológica o química, así como simpatizar con la idea de que un alimento ofrezca posibilidades de prevención o tratamiento de enfermedades. La gente demanda cada vez más alimentos saludables y seguros con la plusvalía de mejorar su estado de salud tanto por el aporte de nutrientes necesarios para su desarrollo y manutención como por la oportunidad de mejorar su salud por contener fitoquímicos que benefician a quienes los consumen (Fernández et al., 2013; Hernández, Serralde, Olguín, Meléndez y Amarante, 2011; Iglesias, Alegre, Salas y Egüez, 2018).

La dimensión económica es la segunda que representa una oportunidad en el caso del maíz, pues la sensibilidad demostrada por las mujeres al cambio económico es alta. Y por último, se colocaron los valores culturales y sociales. Estos resultados concuerdan con el análisis de intensidad de la sensibilidad de los valores: se aprecia una intensidad alta en todas las dimensiones. Asimismo, los valores obtenidos en el sentido de su sensibilidad permite suponer que, quizás por las condiciones sociodemográficas de la ciudad, sus pobladores no consideran un cambio sociocultural importante en su comunidad ni detectan alguna posibilidad de riesgo, salvo las sexagenerias que consideran que el aspecto social es igual de prioritario que las primeras dos dimensiones.

Los resultados de la valoración permiten considerar que el aspecto económico debe adaptarse para garantizar la sustentabilidad del maíz y ofrecer mayores mercados y oportunidades en cuanto al comercio justo del grano; también, la dimensión de valores sanitarios hace viable la oferta del maíz como alimento funcional con beneficios para la salud de quienes lo consumen; estos dos resultados serían los pilares de la propuesta del maíz como alimento funcional sustentable que garantice la defensa plena del patrimonio. 


\section{Revista Iberoamericana \\ de las Ciencias Sociales y \\ Humanísticas}

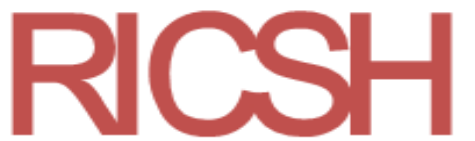

ISSN: $2395-7972$

\section{Conclusión}

Los valores existentes en el uso y consumo del maíz en las mujeres citadinas de San Francisco de Campeche muestran una mayor correlación con la edad. Si bien todas las generaciones exhiben una intensidad alta a la sensibilidad al cambio, las generaciones más jóvenes presentan una menor magnitud en las dimensiones de valores evaluados. Esto representa una urgencia y una necesidad de educar y reeducar a la población respecto a la importancia de la agrodiversidad del maíz y promover sus propiedades para implementar su uso como alimento funcional e impulsar su adherencia a proyectos de desarrollo sustentable y de la defensa del patrimonio.

\section{Referencias}

Altieri, M. A. (2004). Aspectos socioculturales de la diversidad del maíz nativo. Documento preparado para el Secretariado de la Comisión para la Cooperación Ambiental de América del Norte. Recuperado de http://depa.fquim.unam.mx/amyd/archivero/MT_Senado_Aspectos_socioculturales_ maiz_transgenico_M.Altieri_UC_Berkeley_CCA_29082.pdf.

Arellano, H. A. and Arriaga, J. C. (2001). Why Improved Maize (Zea mays) Varieties are Utopias in the Highlands of Central Mexico. Convergencia, 8(25), 255-276.

Brutus, M. D. H. (2007). El Maíz: patrimonio de la humanidad. Correo del Maestro. Revista para Profesores de Educación Básica, (137), 12-18.

Castro, S. G. (2005). El maíz transgénico en México: La contaminación genética de tierras indígenas. Recuperado de https://otrosmundoschiapas.org/wpcontent/uploads/2005/04/ELGMXICO.pdf.

D’Alessandro, R. y González, A. A. (2017). La práctica de la milpa, el ch’ulel y el maíz como elementos articuladores de la cosmovisión sobre la naturaleza entre los tzeltales de Tenejapa en los Altos de Chiapas. Estudios de Cultura Maya, 50, 271-297.

Espinosa, A., López, M. A., Gómez, N., Betanzos, E., Sierra, M., Coutiño, B., Aveldaño, R., Preciado, E. y Terrón, A. D. (2003). Indicadores económicos para la producción y uso de semillas mejoradas de maíz de calidad proteínica (QPM) en México. Agronomía Mesoamericana, 14(1), 105-116. 


\section{Revista Iberoamericana \\ de las Ciencias Sociales y \\ Humanísticas}

ISSN: $2395-7972$

Fernández, R., Morales, L. A. y Gálvez, A. (2013). Importancia de los maíces nativos de México en la dieta nacional: Una revisión indispensable. Revista Fitotecnia Mexicana, 36(supl. 3-a), 275-283.

Garza, A. V. y Cantú, P. C. (2002). Salud ambiental, con un enfoque de desarrollo sustentable. Revista Salud Pública y Nutrición, 3(3).

Hernández, A. A., Serralde, A. E., Olguín, G., Meléndez, G. y Amarante, M. (2011). Medicina y nutrición. Nutrición comunitaria y clínica. México: McGraw-Hill.

Iglesias, S., Alegre, J., Salas, C. y Egüez, J. (2018). El rendimiento del maíz (Zea mays L.) mejora con el uso del biochar de eucalipto. Scientia Agropecuaria, 9(1), 25-32.

Olivé, L. (2009). El maíz en México, problemas éticos-políticos. Ciencias, (92-93), 146-156.

Reynoso, C. R., González, J. E. y Salgado, L. M. (2007). La alimentación del mexicano y la incidencia de diabetes tipo 2. Revista Especializada en Ciencias Químico-Biológicas, 10(1), 36-38.

Rodríguez, T. J., Chávez, M. C., Thomé, H. y Miranda, G. (2017). Elaboración y consumo de tortillas como patrimonio cultural de San Pedro del Rosal, México. Región y Sociedad, 29(70), 155-179.

Sánchez, G. y Cortez, J. (2006). Las identidades cultural y alimentaria en México: un sistema complejo transdisciplinario. Complexus. Revista sobre Complejidad, Ciencia y Estética, (4), 31-57.

Sangerman, D. M., Acosta, J. A., Schwenstesius de Rindermann, R., Damián, M. A. y Larqué, B.S. (2010). Consideraciones e importancia social en torno al cultivo del frijol en el centro de México. Revista Mexicana de Ciencias Agrícolas, 1(3), 363380. 


\section{Revista Iberoamericana de las Ciencias Sociales y Humanísticas}

ISSN: $2395-7972$

\begin{tabular}{|c|c|}
\hline Rol de Contribución & Autor (es) \\
\hline Conceptualización & Rafael Mex Álvarez (principal) Patricia Garma Quen (apoya) \\
\hline Metodología & $\begin{array}{l}\text { Rafael Mex Álvarez (principal) Patricia Garma Quen (apoya) } \\
\text { David Yanez Nava (apoya) }\end{array}$ \\
\hline Software & Rafael Mex Álvarez (principal) David Yanez Nava (apoya) \\
\hline Validación & Rafael Mex Álvarez (principal) David Yanez Nava (apoya) \\
\hline Análisis Formal & $\begin{array}{l}\text { Rafael Mex Álvarez (principal) David Yanez Nava (apoya) } \\
\text { David Yanez Nava (apoya) }\end{array}$ \\
\hline Investigación & $\begin{array}{l}\text { Rafael Mex Álvarez (principal) David Yanez Nava (apoya) } \\
\text { David Yanez Nava (apoya) }\end{array}$ \\
\hline Recursos & $\begin{array}{l}\text { Rafael Mex Álvarez (principal) David Yanez Nava (apoya) } \\
\text { David Yanez Nava (apoya) }\end{array}$ \\
\hline Curación de datos & Rafael Mex Álvarez (principal) Patricia Garma Quen (apoya) \\
\hline $\begin{array}{l}\text { Escritura - Preparación del } \\
\text { borrador original }\end{array}$ & $\begin{array}{l}\text { Rafael Mex Álvarez (principal) Patricia Garma Quen (apoya) } \\
\text { David Yanez Nava (apoya) }\end{array}$ \\
\hline $\begin{array}{l}\text { Escritura - Revisión y } \\
\text { edición }\end{array}$ & Rafael Mex Álvarez (principal) David Yanez Nava (apoya) \\
\hline Visualización & Rafael Mex Álvarez (principal) David Yanez Nava (apoya) \\
\hline Supervisión & $\begin{array}{l}\text { Rafael Mex Álvarez (principal) David Yanez Nava (apoya) } \\
\text { Patricia Garma Quen (apoya) }\end{array}$ \\
\hline $\begin{array}{l}\text { Administración de } \\
\text { Proyectos }\end{array}$ & $\begin{array}{l}\text { Rafael Mex Álvarez (igual) David Yanez Nava (igual) Patricia } \\
\text { Garma Quen (igual) }\end{array}$ \\
\hline Adquisición de fondos & Rafael Mex Álvarez (principal) David Yanez Nava (apoya) \\
\hline
\end{tabular}

